\title{
Developing Therapeutics for the Treatment of Multiple Sclerosis
}

\author{
David J. Virley \\ Neurology and GI Centre of Excellence for Drug Discovery, GlaxoSmithKline Pharmaceuticals, Harlow, Essex CM19 5AW, \\ United Kingdom
}

\begin{abstract}
Summary: Multiple sclerosis (MS) is both a complex and chronic neurological disease of the CNS. This poses unique challenges for drug discovery in terms of delineating specific targets related to disease mechanisms and developing safe and effective molecules for clinical application. Preclinical animal models of MS provide the necessary test bed for evaluating the effects of novel therapeutic strategies. Because the clinical manifestations and pathological consequences of disease vary dramatically from individual to individual, as well as treatment response to existing therapies, this creates a significant research endeavor in terms of translating preclinical methodologies to the clinical domain. Potentially exciting treatments have emerged in the form of natalizumab (Tysabri), an $\alpha 4$ integrin antagonist, and more recently FTY720, a sphinogosine- 1 phosphate receptor modulator, providing a compelling proof-ofprinciple from bench to bedside. However, further research is
\end{abstract}

required to discharge safety concerns associated with these therapeutic avenues. Future prospects in the guise of diseasemodifying therapies that target the inflammatory and neurodegenerative components of disease have come to the forefront of preclinical research with the sole aim of reducing the underlying irreversible progressive disability of MS. Significant progress with novel therapies will be made by implementing biomarker strategies that extrapolate robustly from animal models to the divergent patient populations of MS. The future therapeutic options for MS will depend on improvements in understanding the precise factors involved in disease onset and progression and subsequently the development of oral therapeutics that translate sustained benefit from the preclinical context into clinical reality. Key Words: Multiple sclerosis, inflammation, demyelination, regeneration, experimental autoimmune encephalomyelitis, therapeutics.

\section{INTRODUCTION}

Multiple Sclerosis (MS) is the most common demyelinating disease of the CNS, affecting young adults in their formative years, where current treatments have limited effectiveness. MS is typified pathologically by multiple inflammatory foci, plaques of demyelination, gliosis, and axonal pathology within the brain and spinal cord, all of which contribute to the clinical manifestations of neurological disability. Although the causal events in precipitating the disease are not fully understood, most evidence implicates an autoimmune etiology together with environmental factors, as well as specific genetic predispositions. Functional impairment, disability, and handicap are expressed as paralysis, sensory and cognitive disturbances, spasticity, tremor, lack of coordination, and visual impairment. All these symptoms significantly impact on the quality of life of the individ-

Address correspondence and reprint requests to David J. Virley, Ph.D., Neurology and GI Centre of Excellence for Drug Discovery, GlaxoSmithKline Pharmaceuticals, Third Avenue, Harlow, Essex CM19 5AW, United Kingdom. E-mail: David_J_Virley@gsk.com. ual. The clinical course of MS can vary from individual to individual, but invariably the disease can be categorized into three forms: relapsing-remitting, secondary progressive, and primary progressive. In approximately $85 \%$ of patients with MS, the disease starts with alternating episodes of neurological impairment characterized by relapses with subsequent complete or partial remission. ${ }^{1}$ In the majority of patients over a variable period, this course is followed by a secondary progressive phase where recovery is absent. A minority of patients $(\sim 15 \%)$ display primary progressive characteristics where irreversible worsening of clinical signs manifest from disease onset. ${ }^{1}$ The disease as a whole places a huge burden on economic and societal resources and highlights the importance of developing novel, safe, and effective therapies for MS in treating the underlying and progressive course of the disease.

This article, will review key challenges for drug discovery in MS, based initially on the existing clinical outcome measurements, available preclinical models to simulate the disease process, and treatment response to current therapeutics. Specific emphasis will then be placed on novel therapeutic challenges for MS, drawing 
on the weight of evidence from natalizumab (Tysabri) and examples of novel anti-inflammatory, neuroprotective, and regenerative approaches. Improving the translational quality of candidate compounds from bench to bedside, involving the utility of biomarkers will also be highlighted to help guide the future development of robust treatment options for MS.

\section{EVALUATION OF CLINICAL OUTCOME IN MS}

Due to the fluctuating nature and breadth of symptoms, robust measurement of the clinical manifestations of MS is problematic. For appropriate assessment of efficacy of drug treatment within clinical trials, the measurement tool(s) should be sensitive and reproducible enough to detect a significant treatment effect. The Expanded Disability Status Scale (EDSS) is considered the most widely used instrument to evaluate therapeutic strategies in MS, despite drawbacks of reproducibility and inadequate representation of upper limb function and cognitive decline. ${ }^{2}$ More recent developments by the National MS Society's clinical outcomes task force in MS have provided a more quantitative and sensitive tool in the MS Functional Composite (MSFC). ${ }^{3}$ The MSFC provides more objective measures of leg function, arm and hand function, and cognitive function. This instrument comprises of three specific tests that probe walking speed (timed 25 foot walk), fine upper limb dexterity ( 9 hole peg test), and cognitive processing (paced auditory serial additional test) on a continuous scale. The MSFC has been shown to be more sensitive to change than EDSS, and during and after treatment with the corticosteroid, methylprednisolone, clinical improvements from acute relapses were more consistently measured. ${ }^{4}$ Furthermore, MSFC scores have been found to correlate with EDSS, magnetic resonance imaging (MRI) lesion load, and self-reported quality of life. In terms of application to clinical trials, the MSFC has been reported to be strongly predictive of clinical and MRI status in relapsing-remitting patients and may offer improved sensitivity to assessing progression in the course of disease and ultimately, the effects of novel disease-modifying therapies for MS. Recent concurrent validation of the MSFC with MRI has been established to determine biological sensitivity to disease severity. ${ }^{5}$ In this study, the EDSS was directly compared with the MSFC in relation to MRI measurements of lesion load. The EDSS was not shown to correlate with MRI measures of disease, whereas the MSFC was shown to correlate with both T1 and T2 lesion load, especially in both relapsing-remitting and secondary progressive MS patients. Although MRI provides valuable primary end-points in phase II clinical trials and supportive outcome measures to phase III clinical trials, as a putative surrogate marker of disease ac-

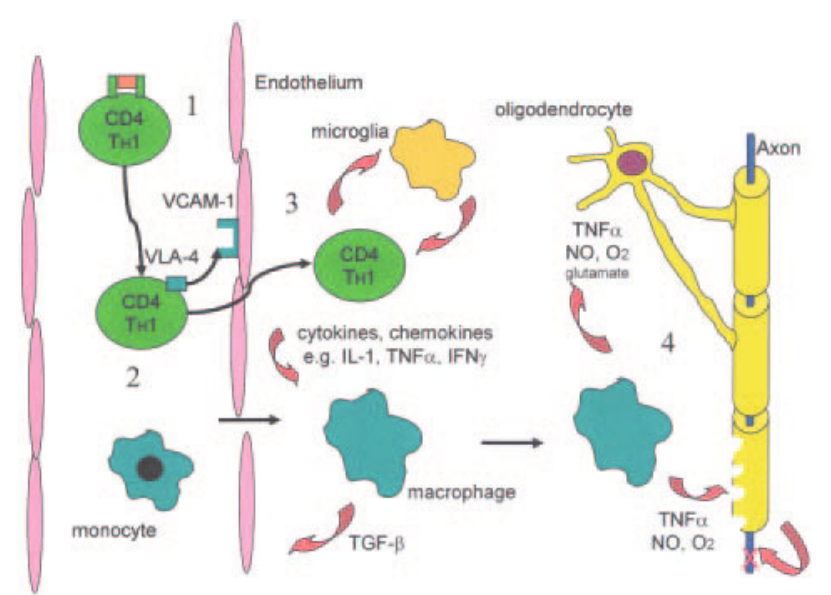

FIG. 1. Schematic view of the putative pathogenic steps in MS. 1: Activation of autoreactive $T$ cells by antigen presenting cells in the periphery. 2: Migration of T cells and monocytes through the blood brain barrier. 3: Amplification of local inflammation and activation of resident microglia. 4: Release of toxic mediators damages myelin and oligodendrocytes with the culmination of axonal loss.

tivity, further developments on assessing additional MRI parameters are required to improve the association with clinical disability. The evolving development of more sensitive, predictive, and practical measures of impairment and disability aligned with more comprehensive and quantitative assessments of MRI tissue signatures in the brain and spinal cord will help evaluate novel therapeutic strategies for MS.

\section{ANIMAL MODELS OF MS}

A major thrust of preclinical research is to identify and validate novel targets within appropriate disease-relevant models that mimic the clinical situation as closely as possible. Animal models form an essential part of the drug development process to assess the validity of the target for therapeutic intervention and provide proof-ofconcept for clinical progression. Although there is no gold standard model of MS, experimental autoimmune/ allergic encephalomyelitis (EAE) models simulate the clinical and pathological hallmarks of MS in various guises and can provide the necessary predictive index for clinical therapeutic application. ${ }^{6}$ EAE is primarily induced by generating T-cell-mediated immunity to CNS antigens and is commonly modeled in rodents (mice, rats, and guinea pigs). The range of autoantigen preparations used to induce EAE range from whole CNS homogenate (spinal cord) to purified protein and peptides. Myelin basic protein (MBP), proteolipid protein, myelin oligodendrocyte glycoprotein (MOG), S100 $\beta$, and glial fibrillary acidic protein as well as specific peptides from respective parent proteins are encephalitogenic in the appropriate host, as the major histocompatability complex (MHC) is one of the major determinants of immune 
responsiveness and disease susceptibility to these selfantigens. The pathogenic autoimmune steps that are thought to initiate and amplify tissue damage in EAE and MS are described in Figure 1. The key steps are: 1) activation of autoreactive $\mathrm{CD} 4^{+} \mathrm{T}$-cells in the periphery to an antigen; 2) transmigration of proinflammatory $\mathrm{T}$ cells and monocytes through the blood brain barrier (BBB); 3) amplification of local inflammation and activation of resident antigen-presenting cells (APCs), such as microglia; and 4) destruction of oligodendrocytes, myelin sheath, and axons culminating in demyelination and axonal pathology. Neurological deficits in rodent EAE models are typically manifested in an ascending manner, beginning with loss of tail tone and progressing to hind limb paralysis, hind and forelimb paralysis, and death. However, the clinical course of EAE is greatly dependent on the type of CNS antigen used, immunization protocols, species and strain of animal used to induce disease. For a valid model of EAE to adequately mimic the clinical condition of acute or chronic progressive MS, enduring pathological signatures such as inflammation, gliosis, oligodendrocyte degeneration, demyelination, and axonal loss should be readily observed within the brain and spinal cord. A number of EAE models possess some but not all these characteristic features, each of which can provide valuable insight into target identification and validation for drug discovery in MS. Depending on the hypothesis being tested for a specific target of interest, the choice of model in the appropriate species allows assessment of the target in the pathological process and the putative mode of action of a therapeutic acting at that specific target. Therefore, a number of rodent EAE models can recapitulate different phases of the disease process such as a rapidly progressing acute monophasic disease, a relapsing-remitting clinical course or a chronic progressive outcome with varying degrees of inflammation, gliosis, oligodendrocyte degeneration, demyelination, gliosis, and axonal pathology in the CNS.

The identification of a target antigen that significantly contributes to clinical severity, lesion topography and the extent of demyelination in animal models of EAE has been attributed to MOG. MOG is a quantitatively minor myelin protein (less than $0.05 \%$ of total myelin proteins), with an Ig-like extracellular domain that is expressed in abundance on the outer most layer of myelin sheaths, which may render it accessible to antibody attack. Autoantibodies against MOG have been shown to enhance demyelination in several EAE models and localized to disintegrating myelin around axons in lesions of acute MS patients on pathological inspection. Furthermore, anti-MOG antibodies have been demonstrated within the peripheral blood and CSF of MS patients, further associating MOG in the pathogenesis of the disease. On the weight of this evidence, MOG (35-55 peptide)-induced
EAE in the C57BL/6 mouse is a robust model of EAE with a chronic clinical course of disease with accompanying pathological hallmarks of inflammation, gliosis, and demyelination. ${ }^{7}$ The consistency of disease incidence and severity is usually maintained with the addition of Bordetella pertussis toxin, which is thought to open BBB and facilitate the entry of autoreactive T-cells primed by MOG. ${ }^{8,9}$ The clinical and pathological signs of MOG-induced EAE are thought to mimic the chronic sustained and progressive phase of MS, particularly relevant to secondary progressive and primary progressive clinical courses of MS.

Due to the majority of MS patients presenting relapsing-remitting symptoms before progressing onto a chronic phase, a number of animal models of EAE have been designed to simulate the more dynamic clinical and pathological features of relapsing-remitting MS. One such model using the Biozzi $\mathrm{AB} / \mathrm{H}$ mouse, ${ }^{10}$ involves the inoculation of homologous spinal cord homogenate (or more specifically MOG peptide) $)^{11,12}$ in adjuvant without the additional use of Bordetella pertussis toxin, and reproducibly induces a chronic relapsing-remitting demyelinating disease. The dynamic chronicity of symptoms is expressed as an acute induction of disease (loss of tail tone and hindlimb paralysis), followed by reduced severity (remission) and then a relapse disease episode. The development of clinical signs in this model are preceded by a loss in weight, whereas remission periods are associated with an increase in body weight, implicating changes in weight as surrogate markers of disease status. Reductions in the degree of inflammation and evidence for remyelination are thought to reflect the remission period in this EAE model, whereas relapses are thought to be indicative of an amplified inflammatory response, gliosis, and demyelination within the CNS.

A key challenge for investigators using rodent EAE models in preclinical drug development for MS is the assessment of neurological deficits in a more sensitive, objective, and quantifiable manner as opposed to the more traditional, qualitative clinical-grading scales. More specific functional measures assessed in rodent EAE models over time, such as hindlimb sensorimotor behavior $^{13}$ and fine motor coordination, may provide a more powerful and sensitive means in extrapolating more closely to the clinical situation (such as the MSFC outcome measure) and provide a more comprehensive assessment of novel therapeutics targeted for MS.

The utility of nonhuman primate EAE models has provided an improved insight into CNS autoimmunity and ensuing pathology due to their close evolutionary relationship with humans. ${ }^{14}$ Nonhuman primate models of EAE have advantages over rodent models in that they simulate more closely the relapsing-remitting and progressive course of disease and have a more sophisticated neuroanatomy, with a greater ratio of white to gray mat- 
ter, similar to humans. Furthermore, monkeys are outbred in nature, unlike rodents which are inbred, making the individual response to EAE more variable, similar to human MS. The use of the common marmoset (Callithrix jacchus), a small new-world monkey, allows a practical and more sophisticated functional and pathological analysis of EAE disease progression, as well as providing essential middle-ground for the development of novel putative therapeutic agents from rodent models to human clinical trials. The incidence of EAE in marmosets immunized with whole myelin, myelin proteins (MOG), recombinant human MOG 1-125 or specifically MOG 14-36 peptide in adjuvant is $100 \%$, with clinical signs following a relapsing-remitting or chronic progressive course. ${ }^{14}$ The pathological hallmarks relating to large foci of demyelination surrounding perivascular infiltrates (inflammation, gliosis, and remyelination) can be readily visualized by serial in vivo MRI in this animal species, providing valuable pathological correlates to human MS. ${ }^{15}$ Clinical signs are usually preceded by weight loss, and include motor weakness, visual defects and paralysis usually scored on a qualitative grading scale. However, a thorough objective characterization of quantitative functional deficits, particularly locomotor activity, fine-motor movement, visuo-spatial neglect and cognitive function, has yet to be interrogated in the marmoset. The EAE model in the marmoset may bridge the gap for novel therapeutic strategies being progressed for clinical trials, such as humanized antibody approaches (e.g., CD40), ${ }^{16}$ and provide definitive MRI surrogate markers of disease activity and treatment response to help guide phase II proof-of-principle clinical trials.

\section{CURRENT THERAPIES FOR MS}

The treatment of MS is still in its infancy with limited therapeutic options, where the main-stay therapies involve the utility of corticosteroid and immunosuppressive interventions. There are currently only five Food and Drug Administration (FDA)-approved treatments for relapsing-remitting MS: two interferon (IFN)- $\beta 1$ a agents (Avonex and Rebif), one IFN- $\beta 1 \mathrm{~b}$ (Betaseron), glatiramer acetate (GA) (Copaxone) and Mitoxanthrone (Novantrone). For patients with secondary progressive MS, cyclophosphamide (Cytoxan) and mitoxanthrone ${ }^{17}$ are prescribed, although provide only modest benefit with significant toxicity. There are currently no available treatment options for primary progressive MS. The main therapeutic options for patients with MS will now be discussed (i.e., corticosteroids, IFN $\beta$, and GA).

\section{Corticosteroids}

Corticosteroid treatment is extensively used in MS for promoting a hastened recovery following a period of an acute attack. ${ }^{18}$ High-dose methylprednisolone, via the intravenous route, is now more popular than oral prednisone, as it provides a stable therapy for MS patients at the onset of an acute relapse. Although short-term therapy has shown benefit to varying degrees, long-term administration is more useful in the treatment and management of relapsing-remitting MS patients. Dramatic improvement in the clinical course of secondary progressive MS has not been shown with corticosteroid treatment. Although the mechanism of action of corticosteroids in MS is not completely understood, evidence from preclinical research has highlighted a number of putative mechanisms: reduction in BBB disruption, an inhibition of the Th1 immune response, a dampening of T-cell migration and the response to antigens, suppression in the expression of adhesion molecules, and protection of oligodendrocytes from cytokine-induced cell death. EAE models have confirmed suppressive actions of corticosteroid treatment on the clinical course of disease and the use of the anti-glucocorticoid, RU 38486 (mifepristone), has been shown to intensify and reverse steroid-induced inhibition of disease. ${ }^{19,20}$ However, the side effects of corticosteroid treatment should not be underestimated. ${ }^{18}$ Short-term treatment can induce transient changes in mood, headache, gastrointestinal pain, and myalgias. Chronic treatment may decrease bone density, leading to osteoporosis with risk of fractures, and infections making the suspension of treatment more appropriate for management of the patient.

\section{IFN- $\beta$}

The IFN- $\beta$-based therapies have been established after 25 years of clinical development. The original rationale for exploring the effects of IFNs in MS was based on the premise that MS was thought to be a virally mediated disease. However, this antiviral hypothesis was untenable based on a clinical trial assessing IFN $\gamma$ where clinical symptoms worsened, suggesting that IFN $\gamma$ played a role in the pathological process of MS. IFN $\beta$, like other IFNs, is a species-specific glycoprotein that has numerous biological properties. Although its mechanism of action is still poorly understood, immunomodulatory as opposed to antiviral and antiproliferative effects seem to predominate. IFN $\beta$-1a is identical to the natural IFN- $\beta$, whereas IFN $\beta$ - 1 b differs by two amino acids and is not glycosylated. Irrespective of these subtle structural differences IFN $\beta-1 \mathrm{~b}$ shows similar biological activity to $\operatorname{IFN} \beta-1 \mathrm{a}$. The putative effects of IFN $\beta$ on MS progression primarily relate to antiinflammatory effects: dampening the stimulatory effects of IFN $\gamma$, tumor necrosis factor (TNF) $\alpha$, interleukin (IL)-12, and lymphotoxin secretion; inhibiting monocyte activation; preventing the disruption of the BBB and thereby reducing the entry of lymphocytes into the CNS; reducing antigen presentation to T-cells; and up-regulation of anti-inflammatory cytokines such as TGF $\beta$ and IL-10. EAE models have dem- 
onstrated that IFN $\beta$ reduces the progression of disease, delays the exacerbation onset and rate, ${ }^{21}$ and may modulate the IL10/IL-12 circuit reducing the effect of epitope spreading and disease severity. ${ }^{22}$

In the clinical trials that have been conducted with both IFN $\beta$ formulations, the key efficacy findings relate to: one third reduction in relapse rate at higher doses; rapid onset of effect, within 1 year for relapse rate and within a few weeks for MRI disease activity; disproportionately large effects on inflammation as measured by MRI activity; slowing of the accumulation of MRI burden of disease; and a tendency for a reduction in the number of patients with observed progression of disability. ${ }^{23-25}$ However, adverse effects are associated with IFN $\beta$ therapy, such as flu-like symptoms and injection site reactions. Discontinuation of IFN $\beta$ therapy is warranted where patients show no improvement over a 6-month period, and where disability progresses or more relapses occur with three or more courses of corticosteroids over a 1-year period. Additional concerns over severe depression or suicidal ideation, drug toxicity, and noncompliance highlight the need for alternative classes of drug with a better therapeutic index. Issues concerning the effects of neutralizing antibodies on IFN $\beta$ efficacy also need to be elucidated in relation to the potential long-term complications for MS patients on $\operatorname{IFN} \beta$ treatment.

\section{GA}

GA is non-IFN, nonsteroidal therapy that constitutes a mixture of synthetic random base copolymers of four amino acids (alanine, glutamic acid, lysine, and tyrosine), in a highly specific molar ratio. Original research investigated the potential encephalitogenic role of GA in animal models of EAE, but unexpectedly GA suppressed the acute and chronic clinical and pathological hallmarks of EAE in a number of animal species. ${ }^{26}$ These effects translated into clinical benefit, in that an initial phase II trial demonstrated GA to reduce relapse rates by $76 \%$ in relapsing-remitting MS patients. ${ }^{27}$ Further clinical development confirmed reductions in relapse rates by a third and a higher preponderance of patients relapse-free. ${ }^{28}$ These effects were confirmed on follow-ups for more than 5 years on treatment and demonstrated sustained efficacy for GA in slowing the progression of disability. Lesion burden assessed by MRI has shown a beneficial profile for GA in relapsing-remitting patients, in that treatment reduced the frequency of new enhancing lesions and lesion load compared to baseline pretreatment measures. ${ }^{29}$ However, no significant improvement in the course of the disease with GA has been demonstrated for secondary progressive MS patients. ${ }^{26}$ A number of mechanisms have been proposed related to its biological activity in relapsing-remitting MS: induction of antigenspecific suppressor $\mathrm{T}$ cells and competitive inhibition of
MBP and related-peptides from antigen-presenting cells. Although GA is well tolerated in MS patients, administration by the subcutaneous route induces localized injection site reactions in the majority. Generally, it is viewed that GA has the most favorable adverse effect profile in that there is a reduced propensity to develop depression, menstrual disorders, neutralizing antibodies compared with the other therapeutic options available for MS. However, there is clear need to develop more improved treatment options for MS patients, which offer sustained relief with greater efficacy without associated risks. This poses a huge challenge for the pharmaceutical and biotechnology industry. A number of alternative disease-modifying strategies will now be presented each of which exert different modes of action and target different phases of the disease process.

\section{EXAMPLES OF NOVEL THERAPEUTIC CHALLENGES FOR MS}

\section{Blockade of lymphocyte migration}

Very late antigen-4: natalizumab (Tysabri) and small molecule antagonists. A key step in the early phase of EAE and MS is the binding of leukocytes to the vascular endothelium of the BBB, before their penetration through it by diapedesis to enter the brain parenchyma (FIG. 1). A substantial body of evidence has now been accumulated that implicates very late antigen- 4 (VLA-4, $\alpha 4-\beta 1$ integrin) in this process, via its interaction with receptors such as vascular cell adhesion molecule 1 (VCAM-1) and the CS1 domain of fibronectin. ${ }^{30,31}$ For example, surface expression of VLA-4 has been shown to be essential for the entry of T-cell clones into the brain, ${ }^{32}$ and in a number of different EAE models treatment with anti-VLA-4 monoclonal antibodies has been effective in suppressing the clinical signs of disease and T cell infiltration into the CNS. ${ }^{32-35}$ Peptide blockers of VLA-4 have likewise been shown to be effective in $\mathrm{EAE},{ }^{36}$ preventing the development of clinical signs and cellular infiltration. ${ }^{37}$ Direct in vivo evidence has suggested that VLA-4 may be important not only in the capture and adhesion of T cells to microvascular endothelium through interaction with VCAM- $1,{ }^{38}$ but also in facilitation of T-cell entry into the brain parenchyma (by the induction of metalloproteinase-2) and in maintenance of the residency of $\mathrm{T}$ cells within the CNS. ${ }^{39}$ However, caution should be exercised based on the preclinical EAE relapsing-remitting model data generated with the PS/2 VLA-4 antibody, demonstrating that, although prophylactic administration suppressed onset and severity of EAE, therapeutic administration at the peak of acute disease or during remission exacerbated disease relapses and increased the accumulation of $\mathrm{CD}^{+}{ }^{+} \mathrm{T}$ cells and VCAM-1 expression in the CNS. ${ }^{35}$ The concerns highlighted by the authors ${ }^{35}$ were that the 
PS/2 VLA-4 antibody could have enhanced costimulatory signals or amplified signals by increased adhesion in the CNS, ultimately augmenting immune responses and clinical severity.

Based on these observations, alternative strategies have been adopted to develop a therapy for MS that is not antibody based. Piraino et al. ${ }^{40}$ recently reported reversal of chronic EAE with a small molecule inhibitor of $\alpha-4$ integrin (CT301), and Cannella et al. ${ }^{41}$ showed efficacy of a synthetic nonpeptide VLA-4 antagonist (TBC 3486) in the acute phase of EAE, but both these agents still had the disadvantage of requiring administration via an injectable route. Furthermore, recent research on highly potent and selective $\alpha 4 \beta 1$ integrin small molecular weight inhibitors, BIO5192 ${ }^{42}$ and 2a-PEG (polyethylene glycol modification of BIO5192), ${ }^{43}$ has demonstrated them to be effective in suppressing EAE with improved pharmacokinetic and pharmacodynamic characteristics. Although these compounds were administered via the subcutaneous and intravenous routes, respectively, the need for less frequent dosing with 2a-PEG at a 30 foldlower dose may provide a reasonable delivery paradigm for therapeutic intervention. ${ }^{43}$ However, the ultimate goal for the future development of small molecule $\alpha 4 \beta 1$ integrin antagonists is oral delivery with improved pharmacological properties that translate into safe and effective interventions for MS.

The clinical significance of VLA-4 in multiple sclerosis has been demonstrated by a 3- to 4-fold increase in its expression on CSF and blood lymphocytes of patients compared with healthy controls. ${ }^{44}$ Encouragingly, clinical treatment with the humanized anti-VLA-4 monoclonal natalizumab (Tysabri), raised against human $\alpha 4 \beta 1$ integrin, significantly reduced the number of new active enhancing lesions seen by MRI over 6 months by about $90 \% .{ }^{45}$ A reduction in lesions was seen in both relapsing and progressive patients, and the therapy also reduced the number of relapses by $50 \% .^{45}$ Although a previous smaller study suggested an increased incidence of relapse following the clearance of the antibody from the circulation, ${ }^{46}$ the subsequent data suggested that there was no rebound effect following drug withdrawal. ${ }^{45}$ These phase II clinical trials complemented the preclinical findings with the murine form of the antibody (AN100266m) before humanization, in that the murine form was a potent inhibitor of in vitro interactions between $\alpha 4 \beta 1$ integrin and VCAM-1, and it suppressed and reversed rodent EAE. ${ }^{47}$ Further clinical trials with natalizumab, involving two randomized, placebo-controlled, double-blind phase III studies were conducted in MS patients who had experienced one relapse in the year before trial enrolment. ${ }^{48}$ The first study demonstrated that after 1 year of treatment with natalizumab monotherapy, the percentage of patients relapse-free was $76 \%$ compared to $53 \%$ in the placebo group. When assessing the MRI end-points $96 \%$ patients on natalizumab treatment had no enhancing lesions, whereas $68 \%$ of patients on placebo had no visible enhancing lesions. The second study showed that patients that had experienced one relapse on IFN $\beta$-1a (Avonex) before trial enrolment were less likely to relapse on natalizumab with Avonex, compared to Avonex with placebo treatment after a study duration of 1 year, i.e., a $54 \%$ reduction in relapse rate. The MRI data also revealed a positive add-on benefit of natalizumab with Avonex, relative to Avonex and placebo, in that $96 \%$ of patients on the natalizumab/Avonex arm showed no enhancing lesions compared to $76 \%$ patients on the Avonex/placebo arm. These results from the 1-year interim analysis were later confirmed after the full 2-year study duration by Elan (Dublin, Ireland) and Biogen IDEC (Cambridge, MA), in that natalizumab reduced the risk of disability progression by $42 \%$ compared with patients on placebo. ${ }^{48}$

Based on the encouraging results from the 1-year interim analysis, the FDA approved natalizumab for the treatment of relapsing-remitting MS. However, on 28 February 2005, 3 months after FDA approval, Biogen IDEC and Elan voluntarily suspended all ongoing clinical trials and marketing of natalizumab. ${ }^{48}$ The reasons for the suspension were that two patients developed progressive multifocal leukoencephalopathy (PML), one fatal, both receiving natalizumab and Avonex for 2 years. ${ }^{49}$ A month later, a third and fatal case of PML was disclosed in patient enrolled in a clinical trial for natalizumab in Crohn's disease. ${ }^{48}$ This information suggested that natalizumab was associated with PML, irrespective of its combination with Avonex and the MS patient population. Although speculation on the likely causes of PML in MS patients in combination with Avonex was fuelled by possible activation of the polyoma JC virus interacting with the antibody for $\alpha 4 \beta 1$ integrin, this hypothesis was not unequivocal based on the case reported in the Crohn's disease trial. ${ }^{48}$ Nevertheless, natalizumab has provided a clear proof-of principle for the $\alpha 4 \beta 1$ integrin target in MS, as preclinical efficacy signals demonstrated in EAE models have translated well into the clinical domain of MS, offering hope as a potential therapeutic application for this debilitating chronic progressive disease. At present, natalizumab and all other VLA-4 antagonist approaches in clinical development are on hold, awaiting a decision from the FDA on the future safety of this drug class involved in blocking lymphocyte migration into the CNS.

\section{Immunomodulatory agents}

FTY720: sphinogosine-1 phosphate modulator. There has been significant interest over recent years toward developing novel immunosuppressive agents for MS. ${ }^{50}$ Immunosuppressants have been used to treat a number of autoimmune diseases (e.g., rheumatoid arthri- 
tis and psoriasis) to reduce disease exacerbations and limit the extent of disease progression. However, immunosuppressant drugs have been associated with significant adverse events, such as renal and liver toxicities, and have been ineffective in the chronic management of disease. Progress has been made recently with FTY720, a synthetic drug produced by a modification to ISP-1 (myriocin: a fungal metabolite), which is thought to act as a sphinogosine-1 receptor modulator. In contrast to the current immunosuppressant agents such as cyclosporin and tacrolimus, FTY720 does not inhibit T-cell activation or proliferation or impair the immunity to systemic viral infection. ${ }^{50}$ Recent evidence from a Lewis rat EAE model has demonstrated that orally administered FTY720 dramatically reduced clinical severity, mortality and the infiltration of leukocytes into the CNS. ${ }^{51}$ Furthermore, Th1 proinflammatory cytokines such as IL-2, IL-6, and IFN $\gamma$ were markedly suppressed in the CNS following prophylactic FTY720 treatment relative to saline treatment. ${ }^{51}$ The mechanistic effect of FTY720 on reducing lymphocyte trafficking and CNS inflammation within EAE, has been interrogated further with contrastenhanced MRI, using superparamagnetic iron-oxide nanoparticles to track macrophage infiltration. ${ }^{52}$ The oral administration of FTY720 was shown to significantly reduce the magnitude and extent of cellular infiltration into the CNS of EAE-sensitized rats. These effects with FTY720 treatment corresponded to reductions in lesion burden and BBB disruption assessed by MRI signatures and a marked reduction in neurological disability during the acute and relapsing phase of the model. Therapeutic administration of FTY720 at the point of relapse also significantly suppressed further progression of clinical signs relative to vehicle treatment, ${ }^{52}$ providing compelling evidence for this novel agent as a potential therapeutic agent for MS. Very recent disclosure of data from a phase II clinical with FTY720, has confirmed a relapse reduction rate of more than $50 \%$ in 281 relapsing-remitting MS patients for 6 months of treatment, relative to placebo. ${ }^{53}$ Inflammatory disease activity, as visualized by gadolinium-enhanced T1-weighted MR imaging was shown to be dramatically reduced by up to $80 \%$, after 6 months of oral, once a day, treatment. ${ }^{53}$ Progressive disease activity in the form of new T2 MRI lesions was also demonstrated to reduce by more than $60 \%$ after FTY720 treatment. ${ }^{53}$ Importantly, the onset of effect was demonstrated as early as 2 months on treatment, and MS patients showed no significant adverse events over the 6-month trial duration relative to placebo. Novartis plan to conduct large scale phase III clinical trials with FTY720 in North America and Europe in the fourth quarter of $2005,{ }^{53}$ the results of which will be eagerly awaited. The current results from phase II proof-of principle trial in MS with FTY720 corroborate the earlier preclinical EAE findings, suggesting a robust predictive translation for a potential novel oral therapeutic for MS.

\section{Anti-inflammatory agents}

COX-2 inhibitors. Cycloxygenase- 1 and -2 (COX-1 and $\mathrm{COX}-2$ or prostaglandin $\mathrm{H}$ synthases 1 and 2) catalyze the conversion of arachidonic acid and oxygen to generate inflammatory prostaglandins such as PGE2, PGD2, PGF2a, and thromboxane TXA2. ${ }^{54} \mathrm{COX}-2$ is ubiquitously expressed in the brain and at the cellular level, is expressed by neurons, microglia and endothelial cells, as well as possibly oligodendrocytes. ${ }^{54} \mathrm{COX}-2$ expression can be highly induced by proinflammatory cytokines, as well as by other factors such as endotoxin, epidermal growth factor (EGF) and TGF (TGF $\beta$ ). Upregulation of COX-2 has also been demonstrated in a number of diseases and/or disease models of the CNS, where an immune-mediated inflammatory response has been linked to a glutamate-mediated excitoxicity. A potential link between COX-2 and inducible nitric oxide (iNOS) has been posited in that both enzymes may act in synergy in inducing excitotoxicy and hence may contribute to the inflammatory, oligogodendrocyte degeneration, demyelination, and axonal pathology witnessed in MS. Furthermore, COX-2 expression is dramatically induced in neurons following ischemic injury, ${ }^{55,56}$ in spinal cords of amyotrophic lateral sclerosis (ALS) patients, ${ }^{57}$ in Alzheimer's disease (AD) brains, ${ }^{58}$ as well as in endothelial cells ${ }^{59}$ and macrophages ${ }^{60}$ within the CNS of EAE rats. Recently, COX-2 expression has also been demonstrated in the brains of MS patients with chronic active lesions (inflammation and demyelination) relative to aged-matched control brains. ${ }^{61} \mathrm{COX}-2$ expression was localized to catabolites of MBP, reflecting recent demyelination, around damaged oligodendrocytes and within immune-derived cells, such as microglia and macrophages. COX-2 expression was also colocalized to iNOS expression, particularly in regions showing active demyelination, suggesting that COX-2 inhibitors could have therapeutic application in MS. As a result of these findings, recently developed COX-2 inhibitors, effective in the treatment of conditions such as inflammatory pain and rheumatoid arthritis, are now being investigated for a broader range of disease indications, including Parkinson's disease, ALS, stroke and AD. ${ }^{62}$

In terms of disease-relevance to MS, nonselective COX inhibitors (BW755c, piroxicam, phenidone), as well as anti-PGE antibodies, have shown prophylactic effects in models of EAE, ${ }^{60,63-65}$ suggesting that the COX-2 component may play a prominent role in the inflammatory pathological cascade of MS. The results from the report, of CNS localization of COX-2 in the brains of MS patients and EAE-sensitized animals, highlights the potential importance of COX-2 in the pathogenesis of MS. Furthermore, the development of potent 
and selective COX-2 inhibitors may provide a novel therapeutic avenue for the treatment of chronic inflammatory conditions such as MS.

\section{Neuroprotective and neuroregenerative therapeutic strategies}

The repair of myelin and neuroprotection signify a major goal for active research in the pursuit of providing long-term benefit and reversing permanent neurological disability associated with MS. ${ }^{66,67} \mathrm{~A}$ number of therapeutic approaches have attempted to limit the extent of oligodendrocyte degeneration, demyelination, and axonal loss and the promotion of remyelination, although none of these strategies have yet been realized clinically. The development of putative neuroprotective treatments for MS has included inhibitors of glutamate excitotoxicity and iNOS, free-radical scavengers, cationic channel blockers etc. ${ }^{67}$ Although efficacy signals have been shown for these targets in a number of EAE models, as well as other neurodegenerative models, proof of principle is still required within the clinical domain of MS. One promising avenue in the form of sodium channel blocker, Flecainide, has recently been shown to reduce the extent of permanent disability and axonal degeneration in a chronic relapsing rat EAE model. ${ }^{68}$ Interestingly, flecainide administration showed protection either before immunization or at the onset of neurological signs, offering potential therapeutic benefit in both relapsing-remitting and progressive forms of MS.

An alternative therapeutic avenue has been to promote axonal regrowth, which in addition to other neurodegenerative conditions such as stroke and traumatic brain and spinal cord injury may also have utility for MS. The existence of myelin inhibitors of axonal growth such as Nogo, myelin-associated glycoprotein, oligodendrocyte myelin glycoprotein and CD100/Sema 4D have offered novel targets for therapeutic development in MS. ${ }^{67} \mathrm{Re}-$ cent evidence for Nogo-66, an extracellular 66 amino acid loop of the Nogo-A protein localized to CNS myelin, has been posited to mediate inhibition of axonal regrowth via interactions with Nogo receptor in EAE. ${ }^{69}$ Nogo KO mice immunized with MOG 35-55 showed a reduction in disease severity and mortality compared to wild-type immunized mice, although histopathological inspection revealed no reductions in lesion area or axonal pathology. ${ }^{69}$ Further interrogation of Nogo 66 as a potential autoantigen in EAE-susceptible strains identified that the immunization of Nogo-66 peptides induced CNS immune responses with clinical and pathological hallmarks of EAE. Specific antibody responses were demonstrated following immunization and marked epitope spreading to other encephalitogenic myelin antigens. However, the authors also noted that some T- and B-cell immune responses to Nogo-66 were associated with suppression of ongoing EAE, suggesting a more compli- cated scenario. ${ }^{69}$ Antibodies against the N-terminal domain of Nogo-A have been recognized in the serum and CSF of MS patients, although the exact role of these antibodies in disease progression or regeneration has yet to be defined. Recent evidence has demonstrated that EAE mice immunized with MOG 35-55 and vaccinated with an anti-Nogo A peptide (623-640) or antibody significantly reduced the onset, severity, and progression of clinical signs together with a marked suppression of inflammation, demyelination and axonal damage/loss in the CNS. ${ }^{70}$ This evidence highlights the potential efficacy of antibodies against Nogo or Nogo-blocking peptides in EAE and the potential application to MS. Trials are currently underway in patients with acute spinal cord injury, assessing the efficacy of anti-Nogo antibodies based on enhanced sprouting responses and functional recovery in both rodent and primate models of spinal cord injury. ${ }^{67}$ Depending on the results from this clinical trial, humanized anti-Nogo antibodies may offer significant progress in the treatment of permanent neurological disability, including the therapeutic indication of MS.

A promising future strategy is to promote myelin repair via targets that promote remyelination or by transplantation of exogenous myelinating cells. Endogenous myelin repair approaches have comprised of growth factor treatments such as PDGF, FGF-2, insulin-like growth factor I (IGF-I), glial growth factor, and neurotrophins. ${ }^{66}$ These targets have been evaluated within a number of in vivo models of nerve injury and EAE and have shown improvements in remyelination and reductions in clinical severity. The most advanced growth factor candidate for MS, IGF-I, has been demonstrated to delay the onset of EAE, although therapeutic administrations at the juncture of established EAE have not yielded positive reductions in the severity of disease. This was corroborated in an open label study where subcutaneous recombinant IGF-I showed no clinical benefit in a small number of MS patients over a 24-week treatment period compared with baseline. ${ }^{71}$ Clearly, the study was underpowered, although future trials investigating the role of growth factor therapy on outcome need to consider the optimal timing for effective and sustained remyelination.

Remyelination by exogenous transplantation has provided a pivotal application for promoting repair in demyelinating diseases such as MS. Preclinical research using a number of in vivo models for various neurodegenerative conditions, in a number of animal species, have confirmed the utility of this therapeutic strategy in restoring neurological function. The proof of principle in MS was evaluated with transplantation of autologous Schwann cells to the lesions of the frontal lobe in a small number of MS patients. ${ }^{66}$ No evidence of survival of grafted cells or signs of remyelination around the lesion area was demonstrated following a biopsy after 6 months. Although the procedure was proven to be safe, 
concerns over survival of transplanted cells that may be susceptible to ongoing disease need to be considered. It should be stressed that the application of focal cell transplantation to treat diseases where there is diffuse multifocal demyelination, as in MS, is a significant hurdle. A possible way forward is the use of multipotent neural precursors or stem cells that have the capacity to migrate to sites of injury and differentiate into neurons, astrocytes and oligodendrocytes to promote regeneration and recovery of function. A pivotal preclinical study that investigated the utility of syngenic adult neural stem cells, derived from the periventrical region of the forebrain of C57 BL/6 mice, demonstrated marked recovery of function and dramatic remyelination in a MOG 35-55 chronic model of EAE. ${ }^{72}$ Stem cells administered either intracerebroventricularly or intravenously were shown to migrate to sites of demyelination and axons undergoing remyelination, and differentiate into oligodendrocytes to

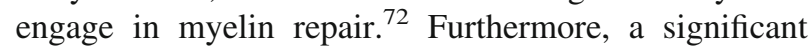
amelioration of astrogliosis, demyelination, and axonal loss was observed in transplanted EAE-sensitized animals. This evidence breaks new ground for cell-based replacement strategies in promoting regeneration to multifocal lesions and restoring loss of function in an EAE model, and hence provides a promising challenge for therapeutic application in MS.

\section{Potential combinations of therapeutic strategies}

Based on the heterogeneity of clinical response and pathological hallmarks ascribed to MS, a pragmatic step would be to combine therapeutic strategies that directly target different aspects of the disease. The crucial development step is to demonstrate that each agent alone provides a benefit of some kind and that the combination should provide a synergistic or additive therapeutic benefit. This may be particularly relevant where patients do not consistently respond to monotherapy and have alternative options available to reduce disease progression. ${ }^{73}$ Drug combinations, with for example IFN $\beta$, that have a distinct mode of action for disease modification (e.g., neuroprotection and regeneration) provide an attractive future therapeutic option for MS patients although methods of evaluation need to be appropriately established to provide a robust meaningful benefit.

\section{FUTURE GOALS FOR DRUG DEVELOPMENT: TRANSLATIONAL SIGNIFICANCE}

For the development of novel therapeutic strategies for MS, it is essential that the disease process is understood more comprehensively and that specific and selective markers are identified to predict clinical course and disease severity. Biomarkers can be used to provide an objectively measured and evaluated indicator of a patho- logical process or a direct pharmacological response to a therapeutic intervention. The use of biomarkers in preclinical animal models and phase I/II proof-of-principle clinical testing will provide a better definition of the mechanism of action related to the candidate drug of interest and help to stratify the proposed patient population of interest for therapeutic efficacy. Furthermore, the utility of a biomarker strategy can not only provide important information on the pharmacokinetic-pharmacodynamic relationship but also help guide dose selection and escalation for compound progression (e.g., lymphocytosis with $\alpha 4 \beta 1$ integrin antagonists). ${ }^{42,43,47}$ For differentiation relative to competitive agents, biomarkers may provide an early opportunity to confirm superiority and compelling efficacy for the therapeutic in question. A number of biomarkers have been proposed for MS, which can be categorized according to specific pathophysiological processes related to disease presentation and progression. ${ }^{74}$ These biomarkers reflect: alteration of the immune system (such as cytokines, chemokines, antibodies, adhesion molecules); BBB disruption; demyelination; oxidative stress and excitotoxicity; axonal and neuronal damage; gliosis; and remyelination and repair. Noninvasive or minimally invasive procedures are crucial for aligning biomarker assessment from the preclinical to clinical setting. In terms of potentially available specimens, these tend to include urine, blood, and CSF measurements for detection of specific markers associated with the disease response or effects of treatment response, although each carry methodological and practical considerations. ${ }^{74}$ Ultimately, biomarkers of disease should have clinical relevance and correlate with disease severity. Although MRI has played an expanding role as a marker of MS disease activity, conventional measures do not necessarily correlate with cumulative functional disability. A number of confounding factors such as disparate locations and sizes of lesions, severity of axonal damage and capacity of remyelination differ among individuals of MS patient. This places huge demands on MRI as a definitive surrogate marker of disease and an index of efficacy for novel therapeutics. Novel MRI signatures of immune-derived disease activity obtained from EAE models, such as the imaging of labeled $\mathrm{T}$ cells with ferumoxides, ${ }^{75}$ may have clinical application in terms of monitoring and categorizing the migration patterns of T-cell phenotypes in the CNS at different stages of disease and confirm immunomodulatory therapeutic strategies of MS, such as FTY720. ${ }^{52}$ Further advanced imaging technologies such as diffusion tensor imaging $(\mathrm{DTI})^{76}$ in both animal models of EAE and MS patients at the level of the brain and spinal cord may offer the opportunity to assess structural alterations that maybe amenable to novel neuroprotective and regenerative therapies. DTI may unlock the key to why the clinical course of MS varies so dramatically and may provide significant 
insight into the mechanisms of disease associated with primary progressive MS.

A systematic approach to biomarker identification will involve comprehensive innovative technologies to identify novel signatures involved the disease process and that respond to change following therapeutic intervention. Novel molecular profiling involving the utility of genomics, proteomics, and metabolomics will help to uncover fingerprints that can facilitate: earlier and more specific diagnoses of MS; identification and validation of therapeutic targets; and monitoring treatment response and ultimately tailoring treatment to specific individuals based on disease course and outcome. ${ }^{77}$ For example, significant insight from gene expression profiling in EAE models have confirmed candidate genes and identified novel targets that contribute to disease severity, offering a potential translational tool for drug discovery. ${ }^{78,79}$ Therefore, novel biomarker identification within preclinical animal models and translation to the clinical setting will permit an improved understanding of the various disease mechanisms in operation and the opportunity to identify and validate novel therapeutic targets for drug development in MS (further information regarding the translational significance of research dedicated to CNS drug discovery is extensively covered in the accompanying article by Ryan and Hurko). ${ }^{80}$

\section{CONCLUSION}

The future offers new and exciting challenges for drug discovery in MS. Significant progress has been made from the preclinical to clinical setting with the emergence of the $\alpha 4 \beta 1$ integrin inhibitor, natalizumab (Tysabri), and more recently the orally active sphingosine-1 phosphate modulator, FTY720; providing novel therapeutic avenues for MS. Markers of safety, however, need to addressed, particularly for VLA-4 antagonists, if these classes of agent are to have any future application in MS. Future alignment of preclinical models with the clinical situation, implementing translational methods and technologies that evaluate the efficacy versus the safety ratios, will improve our understanding of the disease process and ultimately help to define new effective and safe therapeutics for MS.

Acknowledgments: I would like to thank John Davis and Isabel Gonzalez [Neurology and Gastrointestinal (NGI) Centre of Excellence for Drug Discovery (CEDD) at GlaxoSmithKline] for support in writing this manuscript. I would also like to acknowledge all my colleagues within the NGI CEDD at GlaxoSmithKline for providing relentless support in developing and refining models of neurological disease for translation to the clinical situation, with the ultimate objective of developing novel therapeutic strategies for neurodegenerative disorders, such as MS.

\section{REFERENCES}

1. Wingerchuk DM, Lucchinetti CF, Noseworthy JH. Multiple Sclerosis: current pathophysiological concepts. Lab Invest 81:263-281, 2001.

2. Kurtzke JF. Rating neurological impairment in multiple sclerosis: an expanded Disability Status Scale (EDSS). Neurology 33:1444$1452,1983$.

3. Fischer JS, Rudick RA, Cutter GR, Reingold SC. The multiple sclerosis functional composite measure (MSFC): an integrated approach to MS clinical outcome assessment. Mult Scler 5:244-250, 1999.

4. Patzold T, Schwengelbeck M, Ossege LM, Malin JP, Sindern E. Changes of the MS functional composite and EDSS during and after treatment of relapses with methylprednisolone in patients with multiple sclerosis. Acta Neurol Scand 105:164-168, 2002.

5. Kalkers NF, Bergers L, de Groot V, Lazeron RHC, van Walderveen MAA, Uitdehaag BMJ, et al. Concurrent validity of the MS Functional Composite using MRI as a biological disease marker. Neurology 56:215-219, 2001.

6. Gijbels K, Engelborghs S, De Deyn PP. Experimental autoimmune encephalomyelitis: an animal model for multiple sclerosis. Neurosci Res Commun 26:193-206, 2000.

7. Juedes AE, Hjelmstrom P, Bergman CM, Neid AL, Ruddle NH. Kinetics and cellular origin of cytokines in the central nervous system: insight into mechanisms of myelin oligodendrocyte glycoprotein-induced experimental autoimmune encephalomyelitis. J Immunol 164:419-426, 2000.

8. Linthicum DS. Development of acute autoimmune encephalomyelitis in mice: factors regulating the effector phase of the disease. Immunobiology 162:211-220, 1982.

9. Hofstetter HH, Shive CL, Forsthuber TG. Pertussis toxin modulates the immune response to neuroantigens injected in incomplete Freund's adjuvant: induction of Th1 cells and experimental autoimmune encephalomyelitis in the presence of high frequencies of Th2 cells. J Immunol 169:117-125, 2002.

10. Baker D, O'Neill JK, Gschmeissner SE, Wilcox CE, Butter C, Turk JL. Induction of chronic relapsing experimental allergic encephalomyelitis in Biozzi mice. J Neuroimmunol 28:261-270, 1990.

11. Amor S, O’Neill JK, Morris MM, Smith RM, Wraith DC, Groome $\mathrm{N}$, et al. Encephalitogenic epitopes of myelin basic protein, proteolipid protein, and myelin oligodendrocyte glycoprotein for experimental allergic encephalomyelitis induction in Biozzi ABH $(\mathrm{H}-$ $2 \mathrm{~A}^{\mathrm{g} 7}$ ) mice share an amino acid motif. J Immunol 156:3000-3008, 1996.

12. Smith PA, Heijmans N, Ouwerling B, Breij EC, Evans N, van Noort JM, et al. Native myelin oligodendrocyte glycoprotein promotes severe chronic neurological disease and demyelination in Biozzi ABH mice. Eur J Immunol 35:1311-1319, 2005.

13. Buddeberg BS, Kerschensteiner M, Merkler D, Stadelmann C, Schwab ME. Behavioral testing strategies in a localized animal model of multiple sclerosis. J Neuroimmunol 153:158-170, 2004.

14. 't Hart BA, van Meurs M, Brok HPM, Massacesi L, Bauer J, Boon $\mathrm{L}$, et al. A new primate model for multiple sclerosis in the common marmoset. Immunol Today 21:290-297, 2000.

15. 't Hart BA, Vogels J, Bauer J, Brok HPM, Blezer E. Non-invasive measurement of brain damage in a primate model of multiple sclerosis. Trends Mol Med 10:85-91, 2004.

16. 't Hart BA, Blezer ELA, Brok HPM, Boon L, de Boer M, Bauer J et al. Treatment with chimeric anti-human CD40 antibody suppresses MRI-detectable inflammation and enlargement of pre-existing brain lesions in common marmosets affected by MOGinduced EAE. J Neuroimmunol 163:31-39, 2005.

17. Neuhaus O, Kieseier BC, Hartung HP. Mechanisms of mixoxantitrone in multiple sclerosis-what is known? J Neurol Sci 223: 25-27, 2004.

18. Pozzilli C, Marinelli F, Romano S, Bagnato F. Corticosteroids treatment. J Neurol Sci 223:47-51, 2004.

19. Bolton C, Flower RJ. The effects of the anti-glucocorticoid RU38486 on steroid-mediated suppression of experimental allergic encephalomyelitis (EAE) in the Lewis rat. Life Sci 45:97-104, 1989. 
20. Bolton C, Oneill JK, Allen SJ, Baker D. Regulation of chronic relapsing experimental allergic encephalomyelitis by endogenous and exogenous glucocorticoids. Int Arch Allergy Immunol 114:7480, 1997.

21. Yu M, Nishiyama A, Trapp BD, Tuohy VK. Interferon- $\beta$ inhibits progression of relapsing-remitting experimental autoimmune encephalomyelitis. J Neuroimmunol 64:91-100, 1996.

22. Tuohy VK, Yu M, Yin L, Mathisen PM, Johnson JM, Kawczak JA. Modulation of the IL-10/IL-12 cytokine circuit by interferon- $\beta$ inhibits the development of epitope spreading and disease progression in murine autoimmune encephalomyelitis. J Neuroimmunol 111:55-63, 2000.

23. The IFN $\beta$ Multiple Sclerosis Study Group. Interferon $\beta-1 \mathrm{~b}$ is effective in relapsing-remitting multiple sclerosis. I. Clinical results of a multicenter, randomized, double-blind, placebo-controlled trial. Neurology 43:655-661, 1993.

24. The IFN $\beta$ Multiple Sclerosis Study Group and the University of British Columba MS/MRI Analysis Group. Interferon $\beta-1 b$ in the treatment of multiple sclerosis: final outcome of the randomised controlled trial. Neurology 45:1277-1285, 1995.

25. Paty DW, Li DK, University of British Columbia MS/MRI study Group and the IFN $\beta$ Multiple Sclerosis Study Group. Interferon $\beta-1 b$ is effective in relapsing-remitting multiple sclerosis. II. MRI analysis results of a multicenter, randomised, double-blind, placebo-controlled trial. Neurology 43:662-667, 1993.

26. Teitelbaum D, Arnon R, Sela M. Copolymer 1: from basic research to clinical application. Cell Mol Life Sci 53:24-28, 1997.

27. Bornstein MB, Miller A, Slagle S. A pilot trial of Cop 1 in exacerbating-remitting multiple sclerosis. N Engl J Med 317:408 414, 1987.

28. Johnson KP, Brooks BR, Cohen JA. Copolymer 1 reduces relapse rate and improves disability in relapsing-remitting multiple sclerosis: results of phase III multicenter, double blind, placebo-controlled trial. Neurology 45:1268-1276, 1995.

29. Mancardi GL, Sardanelli F, Parodi RC, Melani E, Capello E, Inglese $\mathrm{M}$, et al. Effect of copolymer-1 on serial gadoliniumenhanced MRI in relapsing remitting multiple sclerosis. Neurology 50:1127-1133, 1998.

30. Wayner EA, Garcia-Pardo A, Humphries MJ, McDonald JA, Carter WG. Identification and characterization of the T lymphocyte adhesion receptor for an alternative cell attachment domain (CS-1) in plasma fibronectin. J Cell Biol 109:1321-1330, 1989.

31. Elices MJ, Osborn L, Takada Y, Crouse C, Luhowskyj S, Hemler ME, et al. VCAM-1 on activated endothelium interacts with the leukocyte integrin VLA-4 at a site distinct from the VLA-4/fibronectin binding site. Cell 60:577-584, 1990.

32. Baron JL, Madri JA, Ruddle NH, Hashim G, Janeway CA Jr. Surface expression of $\alpha 4$ integrin by CD4 T cells is required for their entry into brain parenchyma. J Exp Med 177:57-68, 1993.

33. Yednock TA, Cannon C, Fritz LC, Sanchez-Madrid F, Steinman L, Karin N. Prevention of experimental autoimmune encephalomyelitis by antibodies against $\alpha 4 \beta 1$ integrin. Nature 356:63-66, 1992.

34. Kent SJ, Karlik SJ, Cannon C, Hines DK, Yednock TA, Fritz LC, Horner HC. A monoclonal antibody to $\alpha 4$ integrin suppresses and reverses active experimental allergic encephalomyelitis. J Neuroimmunol 58:1-10, 1995.

35. Theien BE, Vanderlugt CL, Eagar TN, Nickerson-Nutter C, Nazareno R, Kuchroo VK, et al. Discordant effects of anti-VLA-4 treatment before and after onset of relapsing experimental autoimmune encephalomyelitis. J Clin Invest 107:995-1006, 2001.

36. Dutta AS, Gormley JJ, Coath M, Hassall L, Hayward CF, Gellert PR et al. Potent cyclic peptide inhibitors of VLA-4 ( $\alpha 4 \beta 1$ integrin)-mediated cell adhesion. Discovery of compounds like cyclo(MePhe-Leu-Asp-Val-D-Arg-D-Arg) (ZD7349) compatible with depot formulation. J Pept Sci 6:398-412, 2000.

37. van der Laan LJW, van der Goes A, Wauben MHM, Ruuls SR, Döpp EA, De Groot CJA, et al. Beneficial effect of modified peptide inhibitor of $\alpha 4$ integrins on experimental allergic encephalomyelitis in Lewis rats. J Neurosci Res 67:191-199, 2002.

38. Vajkoczy P, Laschinger M, Engelhardt B. $\alpha 4$-Integrin-VCAM-1 binding mediates $\mathrm{G}$ protein-independent capture of encephalito- genic T cell blasts to CNS white matter microvessels. J Clin Invest 108:557-565, 2001.

39. Graesser D, Mahooti S, Madri JA. Distinct roles for matrix metalloproteinase-2 and $\alpha 4$ integrin in autoimmune T cell extravasation and residency in brain parenchyma during experimental autoimmune encephalomyelitis. J Neuroimmunol 109:121-131, 2000.

40. Piraino PS, Yednock TA, Freedman SB, Messersmith EK, Pleiss, MA, Vandevert C, et al. Prolonged reversal of chronic experimental allergic encephalomyelitis using a small molecule inhibitor of $\alpha 4$ integrin. J Neuroimmunol 131:147-159, 2002.

41. Cannella B, Gaupp S, Tilton RG, Raine CS. Differential efficacy of a synthetic antagonist of VLA-4 during the course of chronic relapsing experimental autoimmune encephalomyelitis. J Neurosci Res 71:407-416, 2003.

42. Leone DR, Giza K, Gill A, Dolinski BM, Yang W, Perper S, et al An assessment of the mechanistic differences between two integrin $\alpha(4) \beta(1)$ inhibitors, the monoclonal antibody TA-2 and the small molecule BIO5192, in rat experimental autoimmune encephalomyelitis. J Pharmacol Exp Ther 305:1150-1162, 2003.

43. Pepinsky RB, Lee WC, Cornebise M, Gill A, Wortham K, Chen LL, et al. Design, synthesis, and analysis of a polyethelene glycolmodified (PEGylated) small molecule of integrin $\alpha 4 \beta 1$ with improved pharmaceutical properties. J Pharmacol Exp Ther 312: $742-750,2005$.

44. Elovaara I, Ukkonen M, Leppakynnas M, Lehtimaki T, Luomala $\mathrm{M}$, Peltola J, et al. Adhesion molecules in multiple sclerosis: relation to subtypes of disease and methylprednisolone therapy. Arch Neurol 57:546-551, 2000.

45. Miller DH, Khan OA, Sheremata WA, Blumhardt LD, Rice GPA, Libonati MA, et al. The International Natalizumab Multiple Sclerosis Trial Group. A controlled trial of Natalizumab for relapsing multiple sclerosis. N Eng J Med 348:15-23, 2003.

46. Tubridy N, Behan PO, Capildeo R, Chaudhuri A, Forbes R, Hawkins CP, et al. The effect of anti- $\alpha 4$ integrin antibody on brain lesion activity in MS. Neurology 53:466-472, 1999.

47. Rice GPA, Hartung HP, Calabresi PA. Anti- $\alpha 4$ integrin therapy for multiple sclerosis-mechanisms and rationale. Neurology 64:1336-1342, 2005.

48. Steinman L. Blocking adhesion molecules as therapy for multiple sclerosis: Natalizumab. Nat Rev Drug Disc 4:510-518, 2005

49. Adelman B, Sandrock A, Panzara MA. Natalizumab and progressive multifocal leukoencephalopathy. N Engl J Med (10.1056/ NEJMc055235), 9 June 2005.

50. Gonsette RE. New immunosuppressants with potential implication in multiple sclerosis. J Neurol Sci 223:87-93, 2004.

51. Fujino M, Funeshima N, Kitazawa Y, Kimura H, Amemiya H, Suzuki S, et al. Amelioration of experimental autoimmune encephalomyelitis in Lewis rats by FTY720 treatment. J Pharmacol Exp Ther 305:70-77, 2003

52. Rausch M, Hiestand P, Foster CA, Baumann DR, Cannet C, Rudin M. Predictability of FTY720 efficacy in experimental autoimmune encephalomyelitis by in vivo macrophage tracking: clinical implications for ultrasmall superparamagnetic iron oxide-enhanced magnetic resonance imaging. J Magn Reson Imaging 20:16-24, 2004.

53. Multiple sclerosis-FTY720, a novel once-daily oral medication, shows promising results. Medical News Today. Available at www. medicalnewstoday.com. Accessed July 4, 2005.

54. O'Banion MK. Cyclooxygenase-2: molecular biology, pharmacology, and neurobiology. Crit Rev Neurobiol 13:45-82, 1999.

55. Nogawa S, Zhang FY, Ross ME, Iadecola C. Cyclo-oxygenase-2 gene expression in neurons contributes to ischemic brain damage. J Neurosci 17:2746-2755, 1997.

56. Iadecola C, Forster C, Nogawa S, Clark HB, Ross ME. Cyclooxygenase-2 immunoreactivity in the human brain following cerebral ischemia. Acta Neuropathol (Berl) 98:9-14, 1999.

57. Yasojima K, Tourtellotte WW, McGeer EG, McGeer PL. Marked increase in cyclooxygenase-2 in ALS spinal cord-implications for therapy. Neurology 57:952-956, 2001.

58. Ho L, Purohit D, Haroutunian V, Luterman JD, Willis F, Naslund $\mathrm{J}$, et al. Neuronal cyclooxygenase 2 expression in the hippocampal formation as a function of the clinical progression of Alzheimer disease. Arch Neurol 58:487-492, 2001. 
59. Deininger MH, Schluesener HJ. Cyclooxygenases-1 and -2 are differentially localized to microglia and endothelium in rat EAE and glioma. J Neuroimmunol 95:202-208, 1999.

60. Moon C, Alm M, Wie MB, Kim HM, Koh CS, Hong SC, et al. Phenidone, a dual inhibitor of cyclooxygenases and lipoxygenases, ameliorates rat paralysis in experimental autoimmune encephalomyelitis by suppressing its target enzymes. Brain Res 1035:206$210,2005$.

61. Rose JW, Hill KE, Watt HE, Carlson NG. Inflammatory cell expression of cyclooxygenase- 2 in the multiple sclerosis lesion. J Neuroimmunol 149:40-49, 2004.

62. Minghetti L. Cyclooxygenase-2 (COX-2) in inflammatory and degenerative brain diseases. J Neuropathol Exp Neurol 63:901-910, 2004.

63. Weber F, Hempel K. Protection against experimental allergic encephalomyelitis with complete Freund's adjuvant is unaffected by prostaglandin synthesis inhibition. Int Arch Allergy Appl Immunol 89:242-245, 1989.

64. Simmons RD. Hugh AR, Willenborg DO, Cowden WB. Suppression of active but not passive autoimmune encephalomyelitis by dual cyclo-oxygenase and 5-lipoxygenase inhibition. Acta Neurol Scand 85:197-199, 1992.

65. Mertin J, Stackpoole A. Anti-PGE antibodies inhibit in vivo development of cell-mediated immunity. Nature 294:456-458, 1981.

66. Stangel, M. Remyelinating and neuroprotective treatments in multiple sclerosis. Expert Opin Investig Drugs 13: 331-347, 2004.

67. Lubetzki C, Williams A, Stankoff B. Promoting repair in multiple sclerosis: problems and prospects. Curr Opin Neurol 18: 237-244, 2005.

68. Bechtold DA, Kapoor R, Smith KJ. Axonal protection using flecainide in experimental autoimmune encephalomyelitis. Ann Neurol 55:607-616, 2004.

69. Fontoura P, Ho PP, DeVoss J, Zheng BH, Lee BJ, Kidd BA, et al. Immunity to the extracellular domain of Nogo-A modulates experimental autoimmune encephalomyelitis. J Immunol 173:69816992, 2004.
70. Karnezis T, Mandemakers W, McQualter JL, Zheng BH, Ho PP, Jordan KA, et al. The neurite outgrowth inhibitor Nogo A is involved in autoimmune-mediated demyelination. Nat Neurosci 7:736-744, 2004.

71. Frank JA, Richert N, Lewis B. A pilot study of recombinant insulin-like growth factor-1 in seven multiple sclerosis patients. Mult Scler 8:24-29, 2002.

72. Pluchino S, Quattrini A, Brambilla E, Gritti A, Salani G, Dina G. Injection of adult neurospheres induces recovery in a chronic model of multiple sclerosis. Nature 422:688-693, 2003.

73. Kappos L, Kuhle J, Gass A, Achtnichts L, Radue EW. Alternatives to current disease-modifying treatment in MS: what do we need and what can we expect in the future? J Neurol 251 [Suppl 5]:5764, 2004.

74. Bielekova B, Martin R. Development of biomarkers in multiple sclerosis. Brain 127:1463-1478, 2004.

75. Anderson SA, Shukaliak-Quandt J, Jordan EK, Arbab AS, Martin $\mathrm{R}, \mathrm{McF}$ arland $\mathrm{H}$, et al. Magnetic resonance imaging of labeled T-cells in a mouse model of multiple sclerosis. Ann Neurol 55: 654-659, 2004.

76. Clark CA, Werring DJ. Diffusion tensor imaging in spinal cord: methods and applications-a review. NMR Biomed 15:578-586, 2002.

77. Ibrahim SM, Gold R. Genomics, proteomics, metabolomics: what is in a word for multiple sclerosis? Curr Opin Neurol 18:231-235, 2005.

78. Lock C, Hermans G, Pedotti R, Brendolan A, Schadt E, Garren H, et al. Gene-microarray analysis of multiple sclerosis lesions yields new targets validated in autoimmune encephalomyelitis. Nat Med 8:500-508, 2002.

79. Ibrahim SM, Mix E, Bottcher T, Koczan D, Gold R, Rolfs A, et al. Gene expression profiling of the nervous system in murine experimental autoimmune encephalomyelitis. Brain 124:1927-1938, 2001.

80. Ryan J, Hurko O. Translational research in central nervous system drug discovery. NeuroRx 2:671-682, 2005. 